\title{
Accuracy of gadoteridol enhanced MR- angiography in the evaluation of carotid artery stenosis
}

\author{
Fulvio Zaccagna ${ }^{1}$, Beatrice Sacconi ${ }^{1}$, Luca Saba ${ }^{2}$, Isabella Ceravolo ${ }^{1}$, Andrea Fiorelli', lacopo Carbone ${ }^{1}$,
} Alessandro Napoli', Michele Anzidei ${ }^{1 *}$ and Carlo Catalano ${ }^{1}$

\begin{abstract}
Background: To compare image quality and diagnostic performance of Gadoteridol-enhanced MR angiography (MRA) with Gadobutrol-enhanced MRA in the evaluation of carotid artery stenosis.

Methods: MRA was performed in 30 patients with carotid stenosis diagnosed at DUS. Patients were randomly assigned to group A (Gadobutrol-enhanced MRA) or group B (Gadoteridol-enhanced MRA). All examinations were performed with a 3T MR system. Image quality was assessed qualitatively by a 3-grade scale and quantitatively with SNR measurements. Diagnostic performance in the assessment of stenosis, plaque length and morphology was evaluated in the two MRA groups by accuracy calculation and RoC curves analysis using CTA as reference standard. Statistically significant differences in SNR and quality scale were evaluated by the Independent-Samples T Test and Mann-Whitney test, while the Z-statistics was used to compare diagnostic accuracy in the two groups.
\end{abstract}

Results: Image quality was graded adequate to excellent for both GBCAs, without significant differences ( $p=0.165)$. SNR values were not significantly different in group B (Gadoteridol-enhanced MRA) as compared to group A (Gadobutrol-enhanced MRA) (89.32 \pm 70.4 vs $81.09 \pm 28.38 ; p=0.635)$. Diagnostic accuracy was $94 \%$ for the evaluation of stenosis degree and $94 \%$ for the identification of ulcerated plaques in group A, while it was $93 \%$ for the evaluation of stenosis degree and $76 \%$ for the identification of ulcerated plaques in group B, without statistically significant differences ( $p=0.936)$.

Conclusion: No significant difference in terms of image quality and diagnostic accuracy was observed between Gadoteridol-enhanced MRA and Gadobutrol-enhanced MRA in patients undergoing evaluation of carotid stenosis.

\section{Background}

Ischemic stroke is estimated to be responsible for $10 \%$ of all deaths worldwide. It is the second cause of mortality in western countries and the leading cause of mortality in China and Japan [1]. Atherosclerotic disease of the extracranial internal carotid arteries can be recognized as the cause of stroke at least in 25-30 \% of patients [1]. Over the last decade, CT-angiography (CTA) and MRangiography (MRA) have attained a cardinal role in the imaging of carotid arteries thanks to their increasing

\footnotetext{
* Correspondence: michele.anzidei@gmail.com

'Department of Radiological, Oncological and Anatomopathological Sciences, Sapienza - University of Rome, Viale Regina Elena 324, 00161 Rome, Italy

Full list of author information is available at the end of the article
}

diagnostic reliability [2-5]. Since the introduction of contrast-enhanced MRA, several gadolinium-based contrast agents (GBCA) have been tested and implemented in clinical practice [6-9], ranging from standard interstitial molecules to high relaxivity and blood-pool agents $[4,9,10]$. Currently, however, there is no common consensus as to which contrast medium should be used for MRA. Gadoteridol (ProHance ${ }^{\oplus}$, Bracco) is a macrocyclic gadolinium chelate with a recognized low-rate of adverse side effects. Moreover, it is the sole agent approved for high-dose administration $(0.3 \mathrm{mmol} / \mathrm{kg})$ due in part to its high stability and thus lower potential for toxicity from long-term heavy metal deposition [11, 12]. Despite the inherent safety of Gadoteridol, only a few studies have evaluated the performance of this contrast medium in MRA [13-16]. 
The purpose of this study was to prospectively compare the image quality and diagnostic performance of Gadoteridol enhanced MRA with that of Gadobutrol (Gadovist ${ }^{\circ}$, Bayer) enhanced MRA in the evaluation of carotid artery stenosis, using CTA as reference standard.

\section{Methods \\ Patient population}

Between November 2013 and February 2015, 30 consecutive patients (19 men, 11 women; mean age $71.96 \pm 7.6$, age range 51-76 years) with confirmed internal carotid artery (ICA) stenosis were enrolled. Institutional Review Board (IRB) (Sapienza - University of Rome) and patient informed consent were obtained. For a patient to be included confirmation of at least one of the following criteria was needed:

1. Doppler-ultrasonography (DUS) showing a hemodynamically significant stenosis of the internal carotid artery (PSV $125 \mathrm{~cm} / \mathrm{s}$ );

2. Ultrasound examination showing a stenosis of $>30 \%$ according to NASCET criteria [17];

3. Ultrasound examination showing a heterogeneous plaque, irregular surface, intra-plaque hemorrhage or ulceration;

Patients with a general contraindication to MRA/CTA examination and/or with a known allergy to contrast agents and/or laboratory signs of renal failure were excluded from the study.

\section{Imaging technique}

Patients were randomly assigned to group A or B, undergoing MRA after the administration of $0.1 \mathrm{mmol} / \mathrm{kg}$ Gadobutrol $(0.1 \mathrm{~mL} / \mathrm{kg})$ and $0.1 \mathrm{mmol} / \mathrm{kg}$ Gadoteridol $(0.2 \mathrm{ml} / \mathrm{kg})$, respectively; randomization was performed according to a 2:1 ratio with $2 / 3$ of patients undergoing Gadoteridol enhanced MRA.

All examinations were performed on a 3T system (Discovery MR750, GE Healthcare, Milwaukee-WIS, USA; with peak gradient strength $50 \mathrm{mT} / \mathrm{m}$, peak slew rate $200 \mathrm{mT} / \mathrm{m} / \mathrm{ms}$, HD Neurovascular Array configuration 8-channel, 12-element) with the same technical parameters (T1-weighted 3D SPGRE sequence, TR $4.8 \mathrm{~ms}$, TE 1.8 , FA $25^{\circ}$, thickness $0.8 \mathrm{~mm}$, matrix $418 \times 418$ ) in both groups. A contrast agent bolus was administered with an automatic injector at a rate of $1 \mathrm{ml} / \mathrm{s}$ through an 18-gauge cannula placed in the antecubital vein of the right arm, followed by $15 \mathrm{ml}$ of saline solution. The optimal delay between injection and MRA acquisition was visually evaluated by the bolus tracking technique. CTA was performed on a 128-MDCT scanner (Somatom Definition, Siemens Medical System Erlangen, Germany) using a dual-energy protocol ( $80 \mathrm{kV}$ and $140 \mathrm{kV}, 200 \mathrm{mAs}$, pitch 0.8, slice-thickness $1 \mathrm{~mm}$, recon increment $0.9 \mathrm{~mm}$, matrix $512 \times 512$ ). The optimal delay between contrast administration and scan was evaluated with the bolustracking technique, with a region of interest (ROI) placed at the level of the aortic arch and automatic scan triggering with enhancement threshold set at $150 \mathrm{HU}$. The acquisition was performed after administration of $50 \mathrm{ml}$ of nonionic iodinated contrast material (Iomeprol $400 \mathrm{mgI} /$ $\mathrm{ml}$, Iomeron 400, Bracco, Milan, Italy), followed by the injection of $30 \mathrm{ml}$ of saline solution at a rate of $4 \mathrm{ml} / \mathrm{s}$ with the use of a dual-head injector.

\section{Image analysis}

An independent observer (B.S. with 8 years of experience in cardiovascular imaging), blinded to the contrast agent used, assessed the quality of all MRA datasets using a 3point scale (poor: inhomogeneous vessel enhancement, poor intraluminal signal and wall delineation; adequate: homogenous vessel enhancement, sufficient intraluminal signal and wall delineation, motion artifacts that did not impair measurements; excellent: homogenous vessel enhancement, high intraluminal signal, precise wall delineation, no motion artifacts). Quantitative measurements of signal-to-noise ratio (SNR; signal Intensity in the vessel/ Standard Deviation (SD) outside the body) were also performed on each dataset by the same observer. More in detail, in each case a vessel ROI was placed in the terminal common carotid artery just proximal to carotid bifurcation, whereas the background-ROIs were obtained for 3 different images, in ghosts-free areas. Two readers (F.Z. with 9 years' experience and M.A. with 13 years' experience in MRA of the carotid arteries), who were blinded to the contrast agent used, independently evaluated MRA datasets for the presence and degree of steno-occlusive disease (according to NASCET criteria) and plaque characteristics. All image sets were presented in random order to each reader. An independent reader (I.C. with 18 years' experience in cardiovascular imaging) evaluated the stenosis degree and plaque characteristics on CTA images.

\section{Statistical analysis}

Statistical analysis was performed using dedicated software (STATA SE 12 for Macintosh; Stata Corporation; College Station, Texas, USA). The normality of each continuous variable group was tested using the Kolmogorov-Smirnov $\mathrm{Z}$ test. Continuous data are described as the mean value \pm Standard Deviation [95\% confidence interval] or [minimum - maximum] as appropriate; categorical data are expressed as number (percentage). The MannWhitney test was applied to determine significant differences in image quality between the two contrast agents. CTA was used as the reference standard for ROC curve analysis and to calculate sensitivity, specificity, positive predictive value (PPV), negative predictive value (NPV) and 
Table 1 Table shows demographic and clinical characteristics of the groups; categorical data are expressed as number (percentage), continuous data are described as the mean value \pm Standard Deviation [95\% confidence interval] or [minimum - maximum] as appropriate

\begin{tabular}{llll}
\hline & Gadoteridol $(n=21)$ & Gadobutrol $(n=9)$ & $p$ \\
\hline Sex, male & $14(66.7 \%)$ & $5(55.6 \%)$ & 0.57 \\
Age, years & $73.38 \pm 6.4[61-85]$ & $68.56 \pm 9.3[51-81]$ & 0.11 \\
Weight, kg & $71.29 \pm 10.9[53-92]$ & $71.78 \pm 11.4[57-96]$ & 0.91 \\
\hline
\end{tabular}

Differences between the groups were tested using the unpaired t-test and the Mann-Whitney according to the nature of examined data

accuracy for stenosis degree and plaque morphology (i.e. plaque ulceration or surface irregularity); a stenosis of $70 \%$ was used as threshold level for stenosis degree. Areas under the curve (AUC) were compared using the Zstatistic to determine differences in diagnostic performance between the two contrast agents [18]. Pearson's correlation coefficient was used to assess performance in the evaluation of plaque length. Agreement between the two readers was tested using Cohen's kappa test and was deemed poor for kappa values of $0.21-0.40$, fair for values of $0.41-0.60$, good for values of $0.61-0.80$ and excellent for values of $0.81-1.00$ [19]. SNR values were compared using the Independent-Samples T Test. A $p$ value $<0.05$ was considered as statistically significant for all tests used.

\section{Results}

MRA and CTA examinations were successfully performed in all patients, without any adverse reaction related to gadolinium- or iodine-based contrast agent administration. 18 carotid arteries were evaluated in the 9 patients in group A (Gadobutrol-enhanced MRA) and 42 carotid arteries were evaluated in the 21 patients in group B
(Gadoteridol-enhanced MRA) (Table 1). The average contrast agent volume administered was $7.3 \pm 1.2 \mathrm{ml}$ [6-10] in group $\mathrm{A}$ and $14 \pm 1.9 \mathrm{ml}$ [11-18] in group $\mathrm{B}$.

The quality of MRA examination was deemed excellent in $4 / 9(44.4 \%)$, adequate in $4 / 9(44.4 \%)$ and poor in $1 / 9$ (11.1\%) of cases in group A (Gadobutrol-enhanced MRA) and excellent in $15 / 21(71.4 \%)$, adequate in $5 / 21$ (23.8 \%) and poor in 1/21 (4.8\%) of cases in group B (Gadoteridolenhanced MRA), without significant differences (MannWhitney test's $p=0.165)$. The mean SNR values were $81.09 \pm 28.38$ [33.03-151.36] in group A and 89.32 \pm 70.4 [28.3-277.5] in group B, without significant differences (independent-Samples T Test $p=0.635$ ) (Fig. 1).

Diagnostic accuracy in the evaluation of vessel stenosis was 0.94 in group A and 0.93 in group B (sensitivity, specificity, PPV and NPV are reported in Table 2). ROC curve analysis (Fig. 2a and b) demonstrated an AUC of 0.974 \pm 0.028 [95 \% c.i. $0.814-1.0$ ] in group A and $0.969 \pm 0.018$ [95 \% c.i. 0.874-0.999] in group B, without significant differences in the assessment of vessel stenosis (z-statistic $p=0.936)$.

Diagnostic accuracy in the evaluation of plaque morphology was 0.94 in group A and 0.76 in group B (sensitivity, specificity, PPV and NPV are reported in Table 3). ROC curve analysis (Fig. 2c and d) demonstrated an AUC of $0.954 \pm 0.045$ [95 \% c.i. 0.727-0.98] in group $A$ and $0.813 \pm 0.058$ [95 \% c.i. 0.658-0.913] in group $\mathrm{B}$, without significant differences in the assessment of vessel stenosis (z-statistic $p=0.155$ ).

The average plaque length was $12.94 \pm 6.23 \mathrm{~mm}$ [6-20] in group $A$ and $11.9 \pm 7.4 \mathrm{~mm}$ [4-25] in group B. Pearson's correlation coefficient revealed a good correlation between MRA and CTA both for Gadoteridol $(r=$ $0.784 ; p=0.000)$ and Gadobutrol $(r=0.751 ; p=0.000)$.

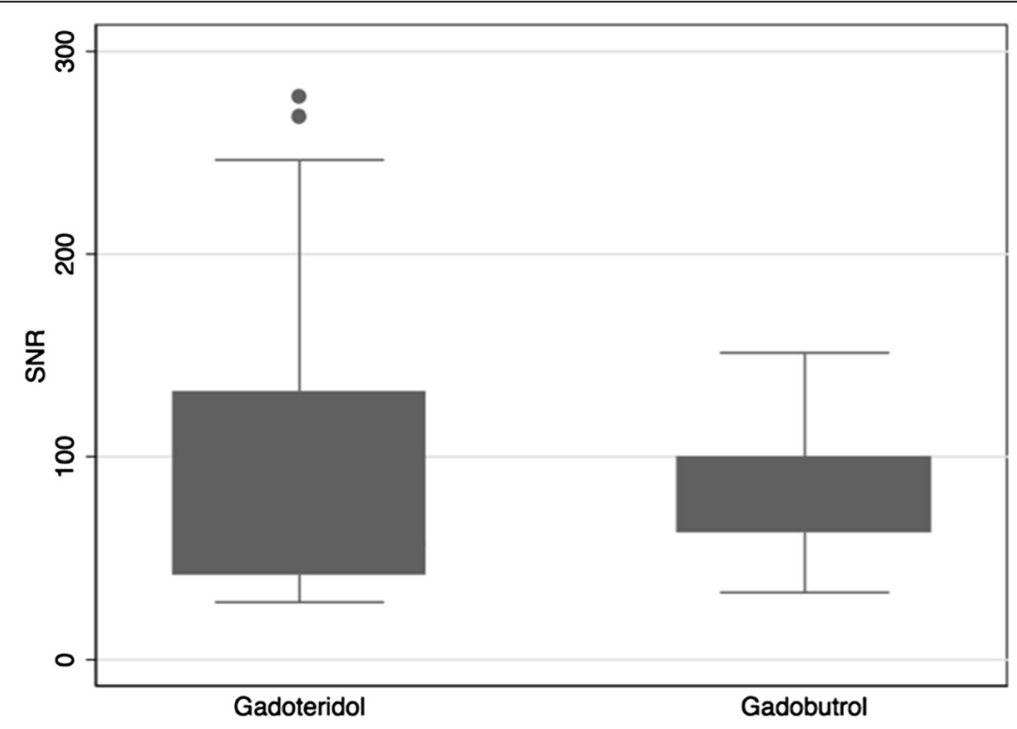

Fig. 1 Figure shows the box plot relative Signal-to-noise ratio according to contrast agents 
Table 2 Table shows accuracy, sensibility, specificity, Positive Predictive Value (PPV) and Negative Predictive Value (NPV) of Gadoteridol-enhanced MRA and Gadobutrol-enhanced MRA in determining stenosis degree

\begin{tabular}{lll}
\hline & Gadoteridol $(n=42)$ & Gadobutrol $(n=18)$ \\
\hline Accuracy & $0.93(39 / 42)$ & $0.94(17 / 18)$ \\
Sensibility & $1(10 / 10)$ & $0.86(6 / 7)$ \\
Specificity & $0.91(29 / 32)$ & $1(11 / 11)$ \\
PPV & $0.77(10 / 13)$ & $1(6 / 6)$ \\
NPV & $1(29 / 29)$ & $0.92(11 / 12)$ \\
\hline
\end{tabular}

Cohen's kappa revealed excellent agreement in stenosis degree evaluation between the two readers $(k=0.861)$ and good agreement in morphology detection $(k=0.733)$ and length determination $(k=0.722)$.

\section{Discussion}

Very few studies have compared Gadoteridol and Gadobutrol for clinical applications [20, 21]. In our studyno significant differences emerge in terms of image quality and diagnostic accuracy between Gadoteridol-enhanced and Gadobutrol-enhanced carotid MRA. Several properties of contrast agents (molecular structure and size, interaction with blood components, concentration) may affect R1 relaxivity and thus influence vascular enhancement, image quality and diagnostic performance in MRA. It has often been claimed that Gadobutrol offers superior image quality in vascular and extravascular applications because of its higher gadolinium concentration $(1 \mathrm{M})$ in the marketed vial as compared with standard contrast agents $(0.5 \mathrm{M})$; however, the clinically approved dose of Gadobutrol is not different from that of other extracellular contrast agents $(0.1 \mathrm{mmol} / \mathrm{kg}$ bodyweight) and, since it belongs to the extracellular class of contrast agents, its relaxivity at standard clinical doses is not affected by the higher concentration [22]. In line with these observations, our results demonstrate that Gadoteridol-enhanced carotid MRA is not inferior to Gadobutrol-enhanced carotid MRA in terms of image quality and diagnostic accuracy. Moreover, the two contrast agents were substantially equivalent in the
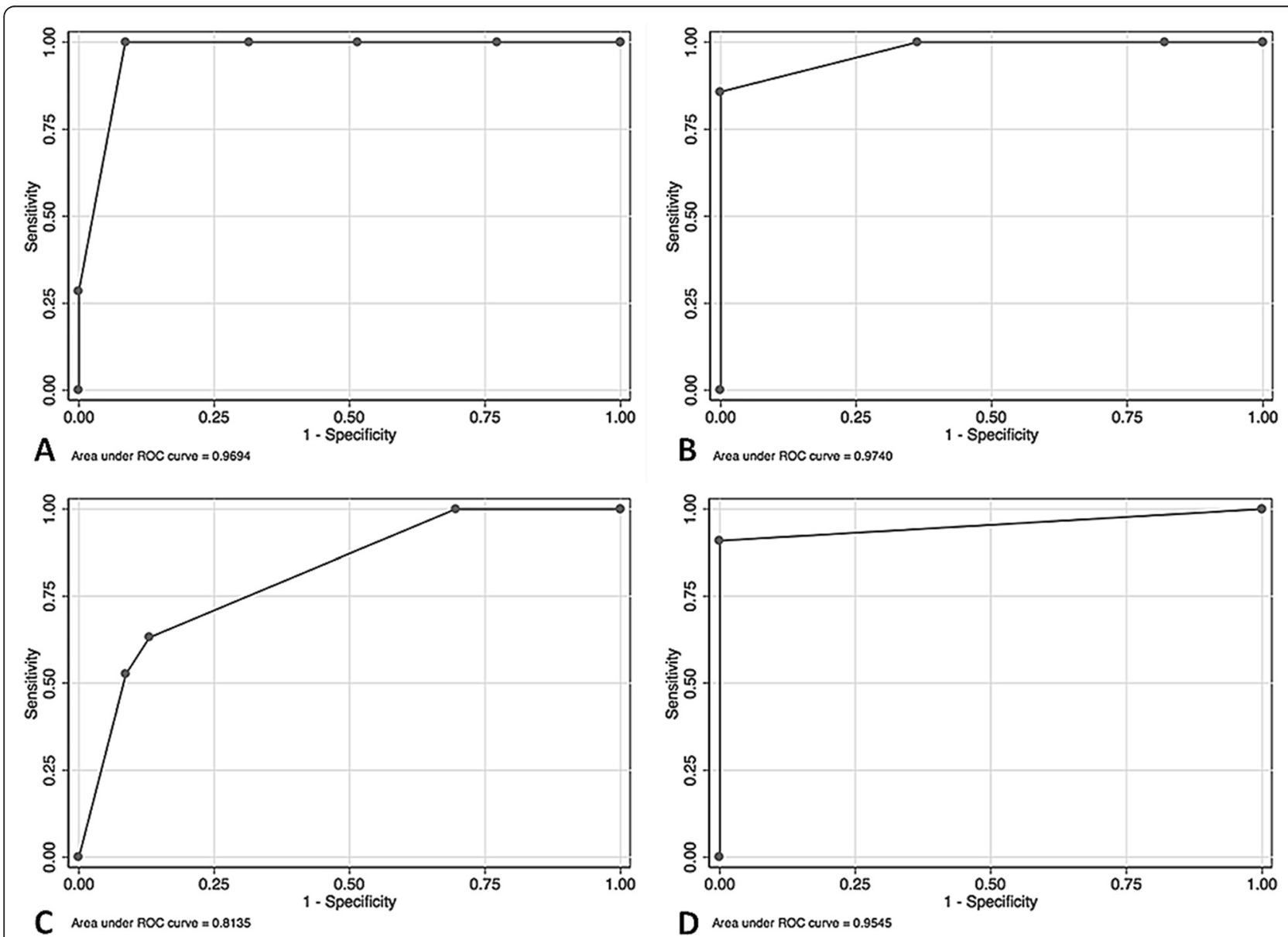

Fig. 2 ROC curves for stenosis evaluation ( $\mathbf{a}$ and $\mathbf{b}$; a for gadoteridol and $\mathbf{b}$ for gadobutrol) and plaque morphology (c and $\mathbf{d}$; $\mathbf{c}$ for gadoteridol and $\mathbf{d}$ for gadobutrol). Area under the ROC curve is showed for each curves 
Table 3 Table shows accuracy, sensibility, specificity, Positive Predictive Value (PPV) and Negative Predictive Value (NPV) of Gadoteridol-enhanced MRA and Gadobutrol-enhanced MRA in plaque morphology evaluation

\begin{tabular}{lll}
\hline & Gadoteridol $(n=42)$ & Gadobutrol $(n=18)$ \\
\hline Accuracy & $0.76(22 / 42)$ & $0.94(17 / 18)$ \\
Sensibility & $0.63(12 / 19)$ & $0.91(10 / 11)$ \\
Specificity & $0.87(20 / 23)$ & $1(7 / 7)$ \\
PPV & $0.8(12 / 15)$ & $1(10 / 10)$ \\
NPV & $0.74(20 / 27)$ & $0.875(7 / 8)$ \\
\hline
\end{tabular}

evaluation of stenosis degree, plaque morphology and length (Figs. 3 and 4). As regards the evaluation of image quality, our results for both subjective assessment and quantitative measurements of SNR, succeed in demonstrating the absence of statistically significant differences in the two groups of patients $(p=0.635)$. It should be noted that high field (3T) scanners, as used in our study, may affect image quality by influencing gadolinium relaxivity and enhancement generated on T1-weighted sequences after intravenous injection in a slightly different manner to that at $1.5 \mathrm{~T}$, at which the majority of contrast agent comparisons have been performed. In particular, at increasing magnetic field strengths gadolinium R1 relaxivity tends to decrease, hence one might expect a parallel deterioration of image contrast in MRA; however, the increased SNR achieved at higher magnetic field, as well as the improved background suppression due to the higher T1 of extravascular tissues, leads to an overall increase of vascular enhancement after contrast agent injection [22]. Even if the relaxivity of both Gadoteridol and Gadobutrol is negatively affected by the increased field strength, the resulting difference remains similar to what has been reported at $1.5 \mathrm{~T}$, with a difference that is not sufficient to significantly influence intravascular enhancement and SNR [6, 23, 24].

The implications of this study for routine practice may be relevant in terms of cost and convenience, since Gadoteridol is less expensive (around 80 euros per single dose vial) in comparison with Gadobutrol (around 130 euros per single dose vial). Moreover Gadoteridol is the sole agent approved for high-dose administration $(0.3 \mathrm{mmol} / \mathrm{kg})$, due in part to its high stability and lower potential for toxicity from long-term heavy metal
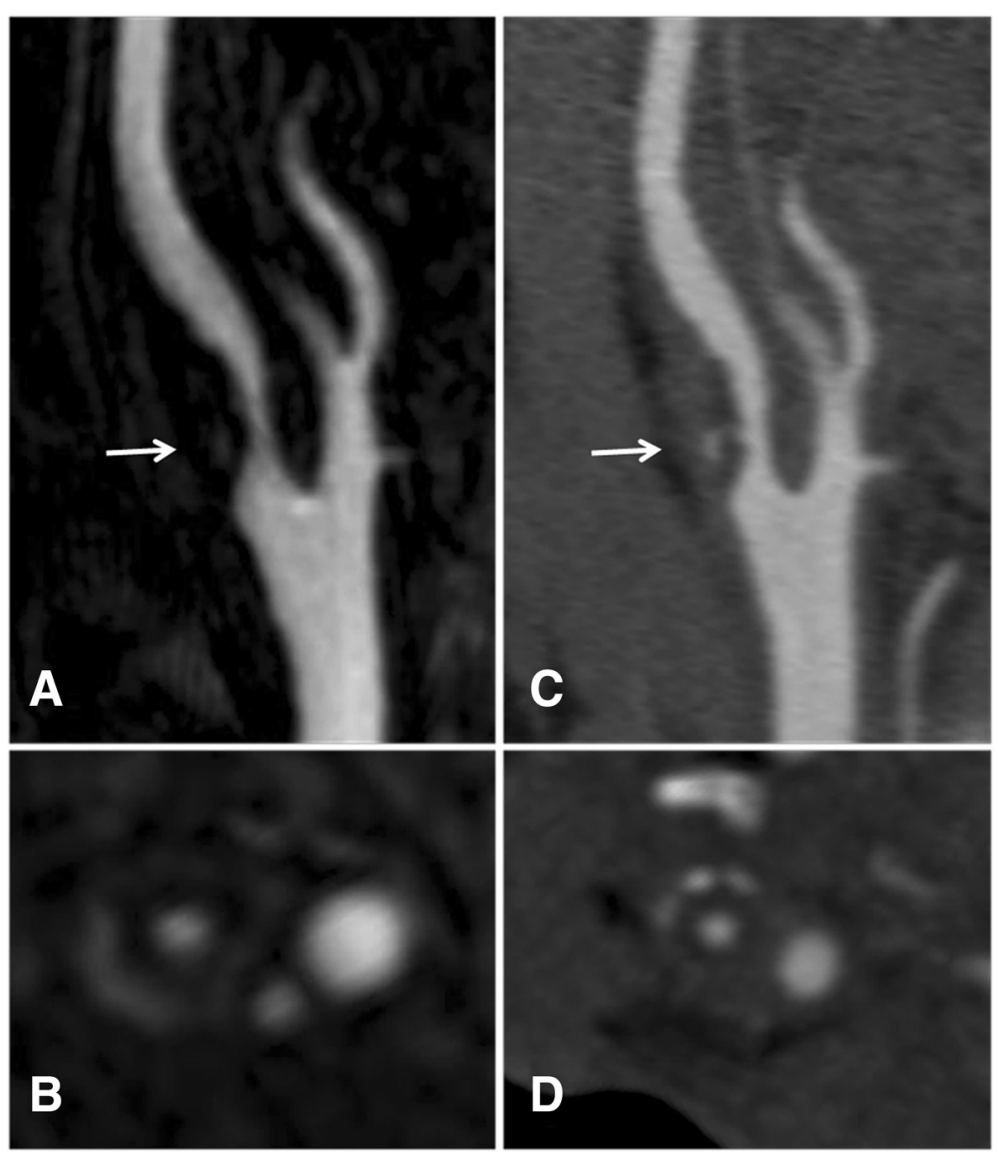

Fig. 3 85-year-old male with tight stenosis of right internal carotid artery (arrow). a and $\mathbf{b}$ CE-MRA images acquired after $0.2 \mathrm{ml} / \mathrm{kg}$ of Gadoteridol, c and d CTA images. CE-MRA demonstrates a ulcerated plaque causing $60 \%$ stenosis of right internal carotid artery; CTA confirms the findings of CE-MRA. Vessel delineation on CE-MRA images was deemed as sharp as on CTA images 

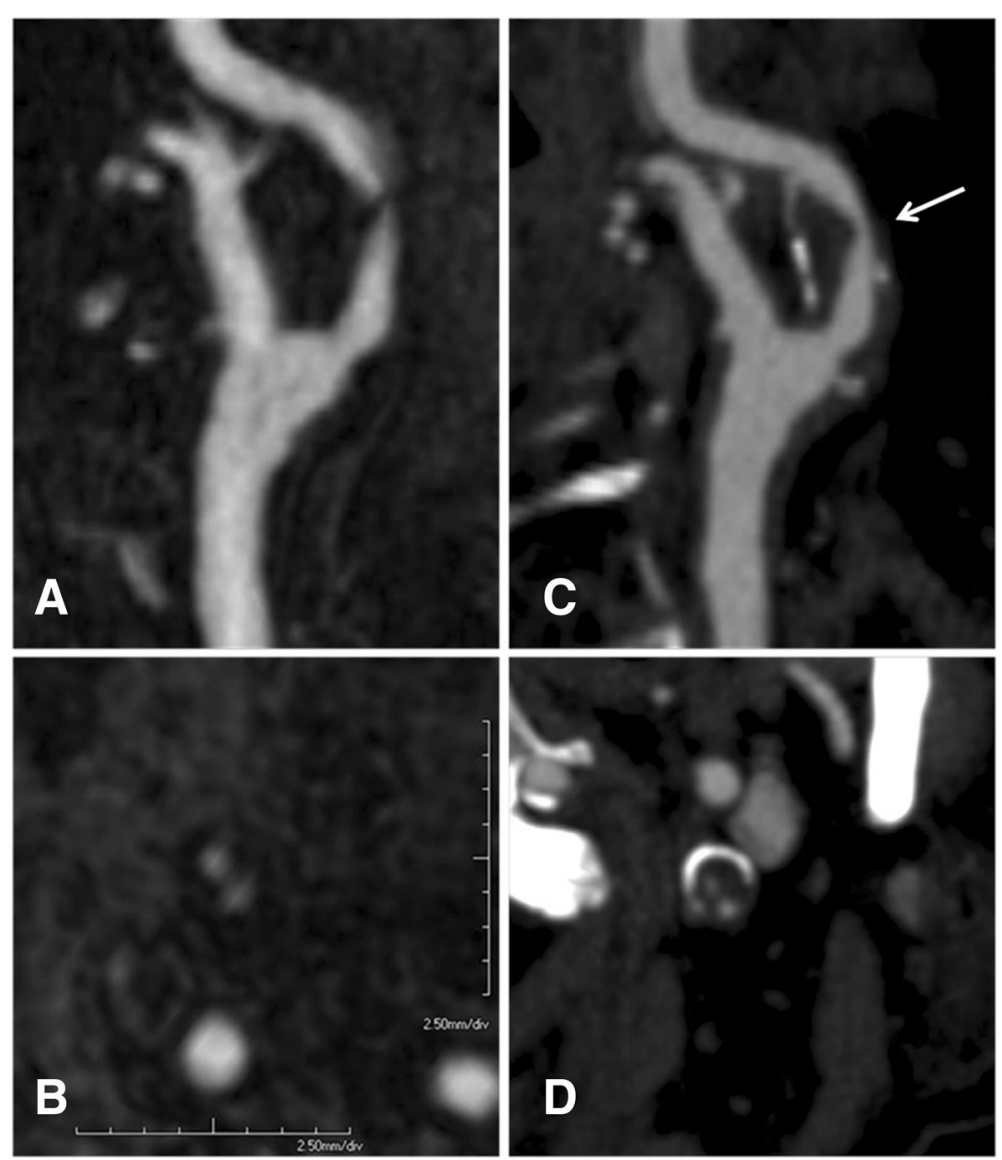

Fig. 4 73-year-old-male referred for carotid imaging to guide revascularization of symptomatic stenosis. a and $\mathbf{b}$ CE-MRA images, c and $\mathbf{d}$ CTA images. Images show an atherosclerotic plaque arising from left carotid arteries bifurcation and involving the proximal segment of internal carotid artery (arrow). Although sagittal-reformatted CE-MRA fails to show residual lumen, axial CE-MRA images demonstrated a severe (90\%) stenosis of left internal carotid artery; CTA confirmed the severe stenosis as well as the absence of ulceration

deposition [10, 11]; this may represent an additional advantage at lower magnetic fields [25]. Furthermore, the adverse effects of Gadoteridol are reported to be very infrequent; it contains a cyclic nonionic chelate and has a high thermodynamic stability constant (23.8) and a long dissociation half-life $(3 \mathrm{~h})$. It's inclusion in the group of agents considered to be at low risk for Nephrogenic Systemic Fibrosis is probably related to these properties [26].

It should be noted that this study has some relevant limitations, the most important being the small sample size; however an extended study with a larger patient population is currently ongoing. In addition, digital subtraction angiography was not available for comparison in our series, since just a minority of patients with clinically significant stenosis underwent stenting procedures while the others were treated with endarterectomy without angiography. However, it should be noted that CTA has been shown to be a valid alternative to DSA as reference standard in comparative studies and, in daily clinical practice, it is currently used as the modality of choice for carotid imaging $[27,28]$. Another relevant limitation is represented by the inter-individual design of the study; however, this was due to ethical reasons since patients with significant disease are referred for treatment rather than a second contrast-enhanced MRA exam. The final limitation concerns the different sizes of the two patient groups; however, unequal randomization has been reported to be an accepted strategy in clinical trials to gain greater experience when comparing a new treatment to a reference standard, hence we adopted this randomization due to the lack of data on Gadobutrol-enhanced carotid MRA (in this regard it is also worth noting that the statistical power of a 1:1 allocation is 0.95 while the statistical power of a 2:1 ratio is 0.925 , with a negligible difference).

\section{Conclusions}

In conclusion, from our study, similar results of Gadoteridol-enhanced MRA compared with Gadobutrolenhanced MRA were observed, both in terms of image quality and diagnostic accuracy, in patients undergoing evaluation of carotid stenosis. 


\section{Competing interests}

The authors declare that they have no competing interests.

\section{Authors' contributions}

SFZ conceived the original study design, carried out the MR examinations, drafted the manuscript, read and approved the final manuscript. BS contributed to the study design, carried out the literature review, drafted the manuscript, read and approved the final manuscript. LS carried out the statistical analysis, drafted the manuscript, read and approved the final manuscript. IC carried out the patients' enrollment, drafted the manuscript, read and approved the final manuscript. AF carried out the MR examinations, drafted the manuscript, read and approved the final manuscript. IC contributed to the study design, drafted the manuscript, read and approved the final manuscript. AN contributed to the study design, drafted the manuscript, read and approved the final manuscript. MA contributed to the study design, carried out the literature review, drafted the manuscript, read andapproved the final manuscript.CC contributed to the study design, drafted the manuscript, read and approved the final manuscript.

\section{Author details}

${ }^{1}$ Department of Radiological, Oncological and Anatomopathological Sciences, Sapienza - University of Rome, Viale Regina Elena 324, 00161 Rome, Italy. ${ }^{2}$ Department of Radiology, Azienda Ospedaliero Universitaria (A.O.U.), di Cagliari-Polo di Monserrato, Italy.

Received: 13 July 2015 Accepted: 15 October 2015

Published online: 15 November 2015

\section{References}

1. Hasso AN, Stringer WA, Brown KD. Cerebral ischemia and infarction. Neuroimaging Clin N Am. 1994;4(4):733-52.

2. Saba L, Mallarini G. Comparison between quantification methods of carotid artery stenosis and computed tomographic angiography. J Comput Assis Tomograf. 2010;34(3):421-30.

3. Clevert DA, Johnson T, Michaely H, Jung EM, Flach PM, Strautz TI, et al. High-grade stenoses of the internal carotid artery: comparison of high-resolution contrast enhanced 3D MRA, duplex sonography and power Doppler imaging. Eur J Radiol. 2006;60(3):379-86.

4. Anzidei M, Napoli A, Marincola BC, Nofroni I, Geiger D, Zaccagna F, et al. Gadofosveset-enhanced MR angiography of carotid arteries: does steady-state imaging improve accuracy of first-pass imaging? Comparison with selective digital subtraction angiography. Radiology. 2009;251(2):457-66.

5. Altaf N, Daniels L, Morgan PS, Auer D, MacSweeney ST, Moody AR, et al. Detection of intraplaque hemorrhage by magnetic resonance imaging in symptomatic patients with mild to moderate carotid stenosis predicts recurrent neurological events. J Vasc Surg. 2008;47(2):337-42.

6. Xing X, Zeng X, Li X, Zhao Q, Kirchin MA, Pirovano G, et al. Contrast-enhanced MR angiography: does a higher relaxivity MR contrast agent permit a reduction of the dose administered for routine vascular imaging applications? Radiol Med. 2015;120(2):239-50.

7. Anzalone N, Scotti $R$, Vezzulli P. High relaxivity contrast agents in MR angiography of the carotid arteries. Eur Radiol. 2006;16 Suppl 7:M27-34.

8. Goyen M, Debatin JF. Gadobenate dimeglumine (MultiHance) for magnetic resonance angiography: review of the literature. Eur Radiol. 2003;13 Suppl 3:N19-27.

9. Amarteifio E, Essig M, Böckler D, Attigah N, Schuster L, Demirel S. Comparison of gadofosveset (Vasovist ${ }^{\oplus}$ ) with gadobenate dimeglumine (Multihance ${ }^{\circledast}$ )-enhanced MR angiography for high-grade carotid artery stenosis. J Neuroradiol. 2014;42(4):236-44.

10. Aime $S$, Caravan P. Biodistribution of gadolinium-based contrast agents, including gadolinium deposition. J Magn Reson Imaging. 2009;30(6):1259-67.

11. Runge VM, Wells JW. Update: safety, new applications, new MR agents. Top Magn Reson Imaging. 1995;7(3):181-95.

12. Shellock FG, Kanal E. Safety of magnetic resonance imaging contrast agents. J Magn Reson Imaging. 1999;10(3):477-84

13. Hathout GM, Duh MJ, El-Saden SM. Accuracy of contrast-enhanced MR angiography in predicting angiographic stenosis of the internal carotid artery: linear regression analysis. AJNR Am J Neuroradiol. 2003;24(9):1747-56.

14. Froger CL, Duijm LE, Liem YS, Tielbeek AV, Donkers-van Rossum AB, Douwes-Draaijer $P$, et al. Stenosis detection with MR angiography and digital subtraction angiography in dysfunctional hemodialysis access fistulas and grafts. Radiology. 2005;234(1):284-91.

15. Hood MN, Ho VB, Foo TK, Marcos HB, Hess SL, Choyke PL. High-resolution gadolinium-enhanced 3D MRA of the infrapopliteal arteries. Lessons for improving bolus-chase peripheral MRA. Magn Reson Imaging. 2002;20(7):543-9.

16. Bremerich J, Colet JM, Giovenzana GB, Aime S, Scheffler K, Laurent S, et al. Slow clearance gadolinium-based extracellular and intravascular contrast media for three-dimensional MR angiography. J Magn Reson Imaging. 2001;13(4):588-93.

17. North American Symptomatic Carotid Endarterectomy Trial Collaborators. Beneficial effect of carotid endarterectomy in symptomatic patients with high-grade carotid stenosis. North American Symptomatic Carotid Endarterectomy Trial Collaborators. N Engl J Med. 1991;325(7):445-53.

18. Hanley JA, McNeil BJ. The meaning and use of the area under a Receiver Operating Characteristic (ROC) curve. Radiology. 1982;143:29-36.

19. Landis JR, Koch GG. The measurement of observer agreement for categorical data. Biometrics. 1977;33(1):159-74.

20. Maravilla KR, Smith MP, Vymazal J, Goyal M, Herman M, Baima JJ, et al. Are there differences between macrocyclic gadolinium contrast agents for brain tumor imaging? Results of a multicenter intraindividual crossover comparison of gadobutrol with gadoteridol (the TRUTH study). AJNR Am J Neuroradiol. 2015;36(1):14-23.

21. Koenig M, Schulte-Altedorneburg G, Piontek M, Hentsch A, Spangenberg P, Schwenke C, et al. Intra-individual, randomised comparison of the MRI contrast agents gadobutrol versus gadoteridol in patients with primary and secondary brain tumours, evaluated in a blinded read. Eur Radiol. 2013:23(12):3287-95.

22. Shen Y, Goerner FL, Snyder C, Morelli JN, Hao D, Hu D, et al. T1 relaxivities of gadoliniumbased magnetic resonance contrast agents in human whole blood at 1.5, 3, and 7 T. Invest Radiol. 2015;50(5):330-8.

23. Hagberg GE, Scheffler K. Effect of $r 1$ and $r 2$ relaxivity of gadolinium-based contrast agents on the T1-weighted MR signal at increasing magnetic field strengths. Contrast Media Mol Imaging. 2013;8(6):456-65.

24. Salvolini U, Scarabino T. High Field Brain MRI: Use in Clinical Practice. 1st ed. Berlin Heidelberg: Springer; 2006.

25. Rinck PA, Muller RN. Field strength and dose dependence of contrast enhancement by Gadolinium- based MR contrast agents. Eur Radiol. 1999;9:998-1004.

26. Reilly RF. Risk for nephrogenic systemic fibrosis with gadoteridol (ProHance) in patients who are on long-term hemodialysis. Clin J Am Soc Nephrol. 2008;3(3):747-51.

27. Anderson GB, Ashforth $R$, Steinke DE, Ferdinandy R, Findlay JM. CT Angiography for the Detection and Characterization of Carotid Artery Bifurcation Disease. Stroke. 2000;31:2168-74.

28. Herzig R, Burval S, Krupka B, Vlachovà I, Urbanek K, Mares J. Comparison of ultrasonography, CT angiography and digital subtraction angiography in severe carotid stenoses. Eur J Neurol. 2004;11(11):774-81.

\section{Submit your next manuscript to BioMed Central and take full advantage of:}

- Convenient online submission

- Thorough peer review

- No space constraints or color figure charges

- Immediate publication on acceptance

- Inclusion in PubMed, CAS, Scopus and Google Scholar

- Research which is freely available for redistribution 